\title{
A Painful Finger: An Unusual Presentation of Von Hippel-Lindau-Associated Advanced Renal Cell Carcinoma
}

\author{
Pui San Sarah Ho Li Yin Yip Mike Nguyen Weeragoda Wijesinghe \\ Arvind Sahu \\ Department of Medical Oncology, Goulburn Valley Health, Shepparton, VIC, Australia
}

\section{Keywords}

Acrometastasis · Von Hippel-Lindau syndrome $\cdot$ Renal cell carcinoma $\cdot$ Metastasis

\begin{abstract}
Bone is reported to be one of the most common sites of metastasis. Acrometastasis is an extremely rare situation and accounts for approximately $0.1 \%$ of all metastatic lesions to the skeleton. Here, we present a case of acrometastasis in a 55-year-old woman who presented with a 3-month history of atraumatic right ring fingertip pain and swelling and was subsequently diagnosed with metastatic renal cell carcinoma. This report highlights the importance of clinical suspicion of malignancy at unusual sites in the setting of non-resolving symptoms.

(C) 2020 The Author(s).

Published by S. Karger AG, Basel

\section{Introduction}

Skeletal metastasis is a common manifestation of advanced malignancy, including renal cell carcinoma. In contrast, acrometastases, metastases located distal to the elbow or knee, are extremely rare. Acrometastasis represents approximately $0.1 \%$ of all bony metastases and renal cell carcinoma accounts for approximately $12 \%$ of these [1]. We report a unique case where acrometastasis was the initial presentation for advanced renal cell carcinoma associated with Von Hippel-Lindau (VHL) mutation.
\end{abstract}

\section{Case Presentation}

A 55-year-old right-hand dominant Caucasian woman presented with a 3-month history of pain and swelling of her right fourth finger. There was no history of trauma. Initial management with antibiotics and steroids did not resolve her symptoms. In addition, the 


\section{Case Reports in \\ Oncology}

1
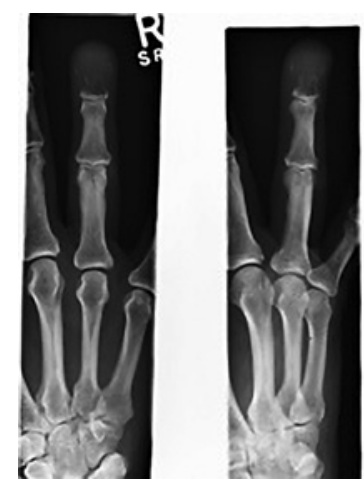

Ho et al.: Acrometastasis in a VHL-Associated Renal Cell Carcinoma

2

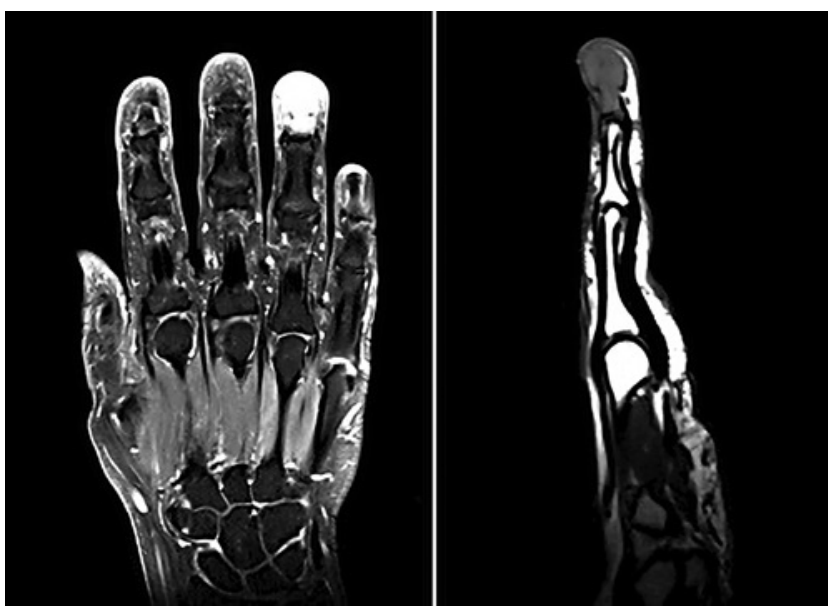

Fig. 1. X-ray of the right hand. Destructive expansile lesion involving the distal end of the terminal phalanx of the ring finger.

Fig. 2. MRI of the right ring finger showing marrow replacement of the distal phalanx of the ring finger by an expansile soft tissue resulting in pathologic fracture.

Fig. 3. Staging CT showing bilateral renal masses, pancreatic cysts and multiple lung metastases.

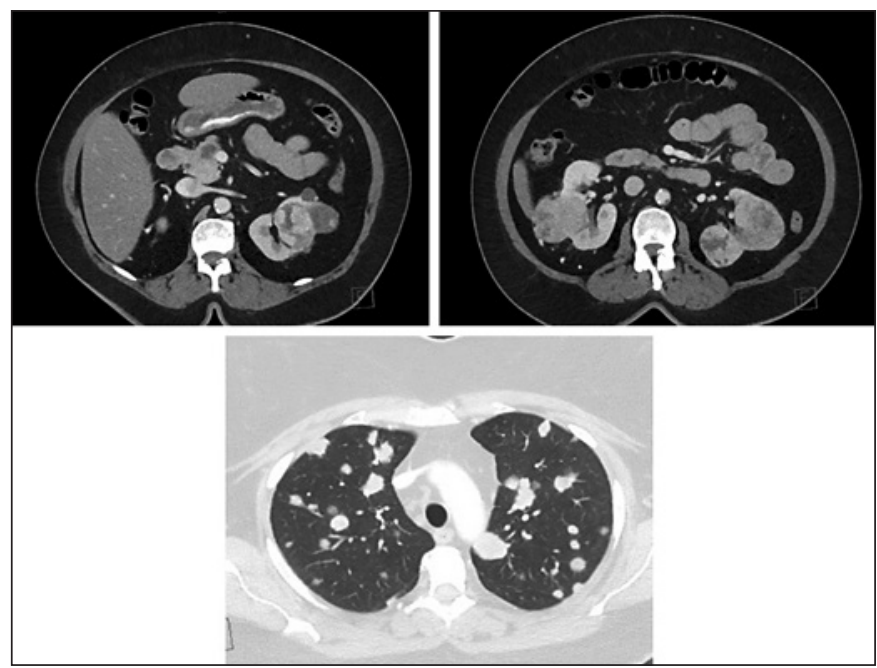

patient had right hip pain which affected her mobility. She also reported to have anorexia, and $10 \mathrm{~kg}$ weight loss over a 3-month duration. Her medical background includes hypertension, hypercholesterolaemia, and type 2 diabetes mellitus, and a 30-pack-year history of smoking. On examination, the distal phalanx of the right fourth finger was swollen and tender on palpation. The range of movement was full at the distal interphalangeal joint but limited by pain.

X-ray of the right hand showed a suspicious lesion involving the distal phalanx of the index finger, which led to further investigations with CT and MRI of the right ring finger, and this confirmed the presence of a destructive expansile lytic lesion involving the distal phalanx (Fig. 1, 2).

Subsequent staging CT of the chest, abdomen and pelvis demonstrated pancreatic cysts, multiple solid-cystic lesions in bilateral kidneys, widespread bilateral pulmonary metastases and widespread bony metastases (Fig. 3). Whole-body bone scan showed osteoblastic metastasis in the left scapula, T5, T6, left acetabulum, and right proximal femur. Biopsy of the distal 
phalanx lesion demonstrated atypical epithelioid and clear cells, consistent with metastatic renal cell carcinoma.

Treatment was commenced with sunitinib, an oral tyrosine kinase inhibitor, as well as palliative radiotherapy to the right hip and thoracic spine. Palliative terminalisation of the right ring finger was performed due to pain and decreased function.

Genetic testing detected a pathologic variant VHL exon 2 mutation. In view of this result, CT of the brain was performed and did not demonstrate any VHL-associated findings and phaeochromocytoma biochemistry testing was unremarkable.

\section{Discussion}

Renal cell carcinoma is a common malignancy with over 350,000 new diagnoses per year worldwide [2]. There is a slight male predominance with a male to female ratio of 1.5:1 and incidence peaks at age $60-70$ years. Unfortunately, $17 \%$ of the cases have distal metastases at the time of diagnosis.

While bony metastases are common in advanced malignancies, these occur most frequently in the axial skeleton and proximal long bones. Acrometastases, metastases distal to the elbow or knee, are very rare and represent only $0.1 \%$ of bone metastases [1]. There is a 2:1 male to female predominance. While involvement of every bone has been described, the third digit is the digit most often affected, and the distal phalanx is the most frequently affected region. Acrometastasis is usually associated with widespread metastatic disease and therefore indicates poor prognosis. The vast majority of cases occur in patients with known metastatic disease with only $10 \%$ of acrometastases thought to be the first sign of malignancy, as occurred in this case [3]. The common underlying primary malignancies include lung, renal cell, breast, and colon [4]. Bone metastases occur from vascular spread, which explains the predilection for marrow-rich bones such as vertebral bodies over acrometastases [5]. Hypotheses that acrometastases more often occur in dominant hands due to increased use and blood supply [6] and that acrometastases are associated with repeated trauma with release of inflammatory cytokines have also been postulated with no significant evidence base [7].

VHL syndrome is an autosomal dominant condition caused by a mutation in the VHL tumour suppressor gene on chromosome 3. VHL gene codes for the VHL protein, which is involved in ubiquitin-dependent proteolysis pathways, hypoxia-inducible factors degradation and subsequent expression of angiogenic and growth factors such as vascular endothelial growth factor [8]. This syndrome predisposes affected individuals to the development of benign and malignant tumours including cysts of the central nervous system, kidney and pancreas, haemangioblastomas, renal cell carcinoma, phaeochromocytoma, neuroendocrine tumours and cystadenomas of the broad ligament and epididymis [9]. The incidence is estimated to be at 1 in 36,000 live births, with a penetrance of over $90 \%$ by the age of 65 . Renal cell carcinoma occurs in $24-45 \%$ of VHL patients with a mean age at presentation of 39 years [10]. VHL syndrome is also associated with renal cysts that are typically multiple, bilateral and occur in up to $63 \%$ of patients.

In summary, this case describes an unusual initial presentation of advanced renal cell carcinoma with acrometastasis and diagnosis of VHL syndrome. While an unlikely diagnosis for a painful, swollen finger, malignancy should be considered as a possible diagnosis in patients with systemic symptoms that do not improve with other therapies. 


\section{Acknowledgement}

The authors would like to acknowledge the contributions of the orthopaedic and palliative care team at Goulburn Valley Health to the management of this patient.

\section{Statement of Ethics}

Ethical review and approval were not required for the study on human participants in accordance with the local legislation and institutional requirements. The patients/participants provided their written informed consent to participate in this study. Written informed consent was obtained from the individual(s) for the publication of any potentially identifiable images or data included in this article.

\section{Disclosure Statement}

The authors declare that the research was conducted in the absence of any commercial or financial relationships that could be construed as a potential conflict of interest.

\section{Author Contributions}

All authors listed have made a substantial, direct and intellectual contribution to the work, and approved it for publication.

\section{References}

1 Flynn CJ, Danjoux C, Wong J, Christakis M, Rubenstein J, Yee A, Yip D, Chow E. Two cases of acrometastasis to the hands and review of the literature. Curr Oncol. 2008 0ct;15(5):51-8.

2 Capitanio U, Montorsi F. Renal cancer. Lancet. 2016 Feb 27;387(10021):894-906.

3 Abrahams TG. Occult malignancy presenting as metastatic disease to the hand and wrist. Skeletal Radiol. 1995 Feb;24(2):135-7.

4 Stomeo D, Tulli A, Ziranu A, Perisano C, De Santis V, Maccauro G. Acrometastasis: a literature review. Eur Rev Med Pharmacol Sci. 2015 Aug;19(15):2906-15.

5 Kerin R. The hand in metastatic disease. J Hand Surg. 1987 Jan;12(1):77-83.

6 Healey JH, Turnbull AD, Miedema B, Lane JM. Acrometastases. A study of twenty-nine patients with osseous involvement of the hands and feet. J Bone Joint Surg Am. 1986 Jun;68(5):743-6.

7 Tolo ET, Cooney WP, Wenger DE. Renal cell carcinoma with metastases to the triquetrum: case report. J Hand Surg. 2002 Sep;27(5):876-81.

8 Lonser RR, Glenn GM, Walther M, Chew EY, Libutti SK, Linehan WM, et al. von Hippel-Lindau disease. Lancet Lond Engl. 2003 Jun 14;361(9374):2059-67.

9 Varshney N, Kebede AA, Owusu-Dapaah H, Lather J, Kaushik M, Bhullar JS. A review of von Hippel-Lindau syndrome. J Kidney Cancer VHL. 2017;4(3):20-9.

10 Ashouri K, Mohseni S, Tourtelot J, Sharma P, Spiess PE. Implications of von Hippel-Lindau syndrome and renal cell carcinoma. J Kidney Cancer VHL. 2015 Sep 25;2(4):163-73. 